\title{
HLA-E expression constitutes a novel determinant for ALL disease monitoring following hematopoietic stem cell transplantation
}

\author{
Sarah B. Reusing ${ }^{1,2}$ - Angela R. Manser ${ }^{1}$ Stefanie Groeneveld-Krentz $\mathbb{1}^{3}$ - Vera Rebmann ${ }^{4}$ - Peter A. Horn ${ }^{4}$. \\ Roland Meisel $\mathbb{D}^{5} \cdot$ Leonid Karawajew ${ }^{3} \cdot$ Arndt Borkhardt $^{2} \cdot{\text { Markus Uhrberg } \mathbb{D}^{1}{ }^{1} \text {. Florian Babor }}^{2}$
}

Received: 28 September 2020 / Revised: 18 January 2021 / Accepted: 28 January 2021 / Published online: 3 March 2021

(c) The Author(s) 2021. This article is published with open access

\section{To the Editor:}

Clinical and experimental evidence suggests that acute lymphoblastic leukemia (ALL) is highly resistant to natural killer (NK) cell-mediated killing, even in the allogeneic setting [1,2]. The mechanisms mediating this resistance are poorly understood but are thought to involve engagement of inhibitory killer immunoglobulin-like receptors (KIR) by HLA-B and HLA-C-encoded ligands, as well as the inhibitory interaction of the NKG2A receptor with HLA-E on leukemic cells [3-5]. Paradoxically, we have recently shown that HLA-E surface expression is specifically downregulated on ALL blasts at the time of diagnosis, whereas residual non-leukemic B cells maintained normal HLA-E expression levels [6].

In order to further explore the diagnostic relevance of HLA-E as a novel marker for disease monitoring, we

These authors contributed equally: Markus Uhrberg, Florian Babor

Supplementary information The online version contains supplementary material available at https://doi.org/10.1038/s41409021-01231-y.

\section{Florian Babor}

Florian.Babor@med.uni-duesseldorf.de

1 Institute for Transplantation Diagnostics and Cell Therapeutics, Heinrich Heine University, Düsseldorf, Germany

2 Department of Pediatric Oncology, Hematology and Clinical Immunology, Center for Child and Adolescent Health, Medical Faculty, Heinrich-Heine University, Düsseldorf, Germany

3 Department of Paediatric Oncology/Hematology, Charité Universitätsmedizin Berlin, Berlin, Germany

4 Institute for Transfusion Medicine, University Hospital Essen, Essen, Germany

5 Division of Pediatric Stem Cell Therapy, Clinic for Pediatric Oncology, Hematology and Clinical Immunology, Center for Child and Adolescent Health, Medical Faculty, Heinrich-Heine University, Düsseldorf, Germany followed the expression of HLA-E through serial timepoints during the disease history of pediatric ALL patients. We started by retrospectively analyzing a cohort of 23 patients diagnosed with precursor B-ALL and for whom samples had been collected at the time of diagnosis, during and after chemotherapy (Supplementary Table S1). In accordance with our previous study, HLA-E downregulation was detected in all patients on leukemic blasts at the time of diagnosis (Fig. 1a, b, and f) but not on residual B cells (data not shown). In patients achieving complete remission following the induction phase $(n=17)$ HLA-E levels were restored to normal ranges and stayed within this range during the observation time (Fig. 1a, f), reflecting the absence of leukemic blasts. In all cases of relapse $(n=6)$, restored HLA-E levels after the induction phase dropped significantly indicating relapse (Fig. 1b, f). As expected, individuals homozygous for genotype $* 01: 03$ showed the strongest HLA-E surface expression. This held true in healthy individuals and in currently and recently [6] investigated ALL patients (Suppl. Fig. S1). Interestingly, downregulation of surface HLA-E was not accompanied by an increase of plasma soluble (s)HLA-E levels, which remained comparable to healthy individuals (Suppl. Fig. S2). In contrast, there was a significant correlation between the percentage of blasts and the downregulation of HLA-E ( $p=0.046$, Suppl. Fig. S3).

We next evaluated HLA-E surface expression in 19 patients undergoing hematopoietic stem cell transplantation (HSCT). Initial values were within the range of the respective stem cell donors (data not shown). Again, patients who achieved long-term remission following HSCT $(n=8)$ stably maintained HLA-E expression levels comparable to initial values (Fig. 1c, f). In contrast, HLA-E expression dropped significantly in relapsed patients, again reflecting recurrence of leukemic blasts $(n=11)$. Among these, six patients with late relapse ( $>6$ months after HSCT) managed to reach reconstituted HLA-E levels initially after HSCT but eventually experienced HLA-E downregulation 
a

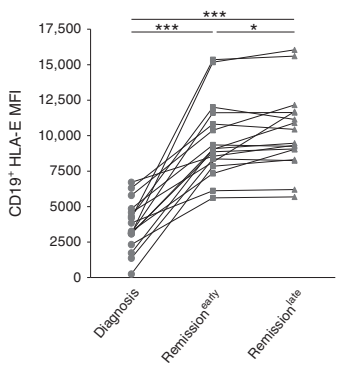

b

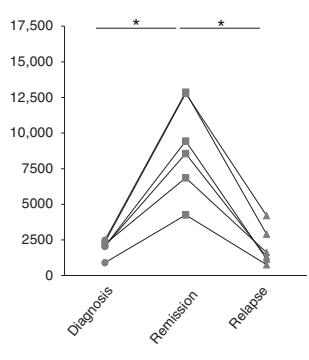

f

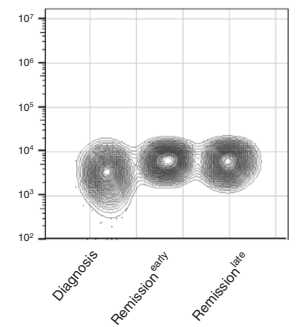

C
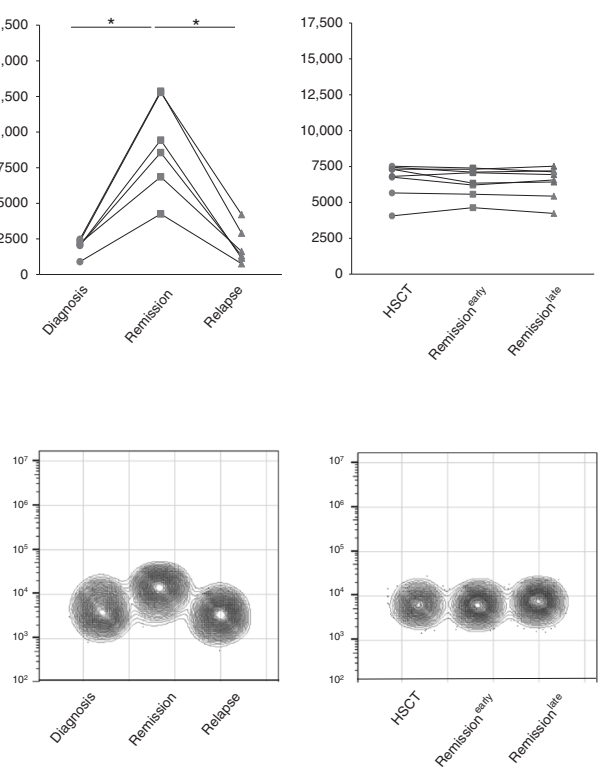
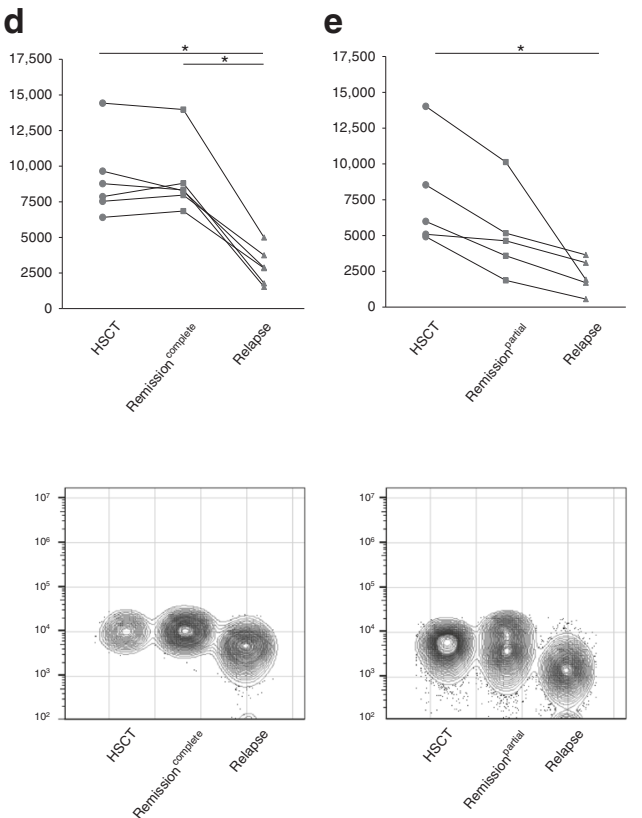

Fig. 1 Consistent downregulation of HLA-E among patients with relapse following chemotherapy or transplantation. Leukemic $B$ cells were stained with HLA-E specific human monoclonal antibody (3D12) and identified via CD19 expression and side scatter. Measurements of all time points were made with identical instrument settings. a HLA-E expression on B cells $\left(\mathrm{CD} 19^{+}\right.$subset) in individual patients treated with chemotherapy without or (b) with relapse at indicated time points (time from diagnosis to early remission 443-826 days, time from diagnosis to late remission 980-1691 days),

at the time of relapse (Fig. 1d, f). Finally, five patients who relapsed early (<6 months after HSCT) showed rapid HLAE downregulation during the partial remission phase followed by a further drop accompanying relapse (Fig. 1e, f).

In order to address the functional relevance and diagnostic potential of the observed HLA-E downregulation, we next studied the cases of three ALL patients undergoing two consecutive HSCTs in more detail (Fig. 2a-d). HLA-E levels remained stable for 178-372 days post 1st HSCT. During this period, minimal residual disease (MRD) levels in BM and PB, measured by PCR $\left(<1 \times 10^{-3}\right)$ and by flowcytometry [7] $(0.00 \%)$ suggested a stable molecular remission. Notably, a subsequent decrease of HLA-E expression occurred in parallel to an increasing MRD load of $\geq 10^{-3}$ (PCR) and $>0.01 \%$ (flow-cytometry), marking the onset of molecular relapse. Once having achieved a further remission, similar impacts on cell surface expression could be observed: following 2nd HSCT, HLA-E levels dropped significantly accompanied by increasing MRD loads. These observations appeared to be independent of the conditioning regimen, donor type, graft-versus-host disease (GvHD) prophylaxis, and presence of GvHD. Furthermore, the correlation was also independent of the HCMV status of the patients (Table S1). (c) HLA-E expression on B cells $\left(\mathrm{CD} 19^{+}\right.$subset) in patients in remission after transplantation. d HLA-E expression on B cells (CD19 ${ }^{+}$ subset) in patients following transplantation with relapse following complete remission or (e) relapse following incomplete remission. $\mathbf{f}$ Flow cytometric data (concatenate contour plots) are shown for representative leukemic patients included in panels a-e, respectively. Statistical significance was determined by one-way-Anova or paired $\mathrm{t}$ test $(* P<0.05, * * * P<0.001)$.

In the next step, we wanted to address the question of whether HLA-E downregulation on peripheral leukemic blasts reflects the degree of expression on immature precursor B cells found in BM samples or is a leukemia-specific mechanism, possibly in order to escape immune surveillance. For this purpose, we assigned each ALL to a specific stage of B cell development according to the expression of stage-specific markers [8] and directly compared their HLAE levels to those of the respective healthy precursor B cell stages found in BM samples (Fig. 2e). First of all, peripheral ALL blasts exhibited a similar degree of HLA-E downregulation compared to ALL blasts found in BM. More importantly, leukemic HLA-E levels were significantly lower than those of the respective healthy precursor B cell stages in BM (Fig. 2e). In the next step, we distinguished in $\mathrm{BM}$ between mature and immature B cells (early precursor hematogones), and leukemic blasts within patient samples by staining for flow-cytometry MRD [7] (Fig. 2f). We found that the HLA-E surface expression on early precursor B cells was significantly lower than on more mature cells. In comparison, the lowest values for HLA-E could be found on leukemic blasts. The data thus suggest leukemia-specific downregulation of HLA-E expression on blasts independent of the underlying B cell precursor maturity stage. 
a

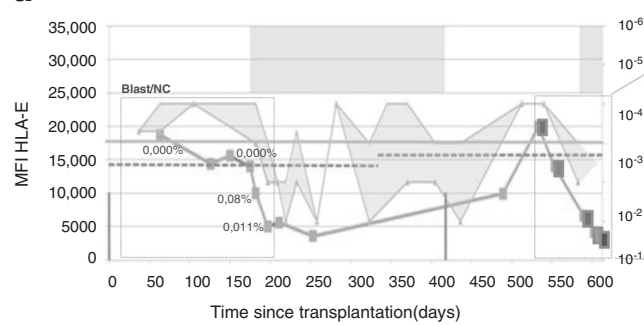

C

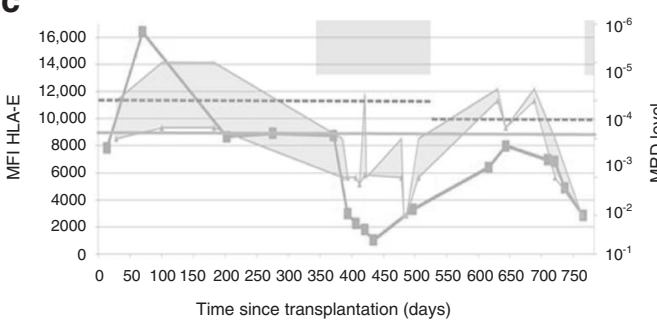

e

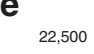

22,500
20,000

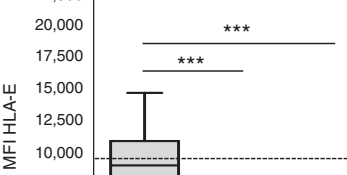

7500
5000

2500
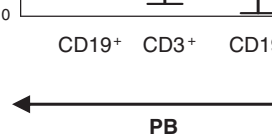

PB

$\mathbf{f}$

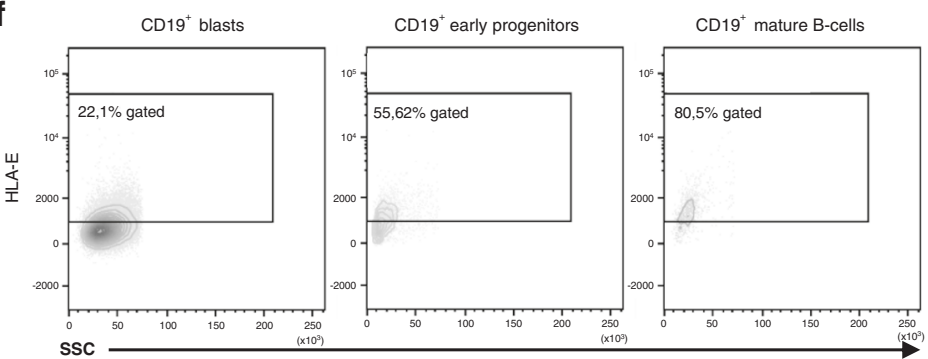

g

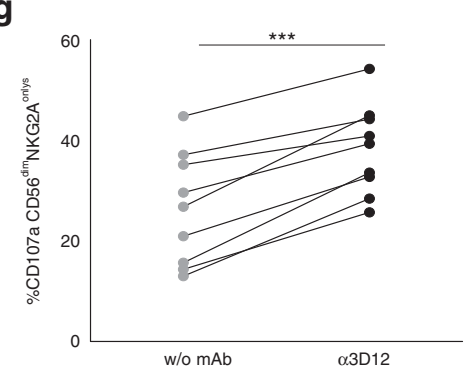

h

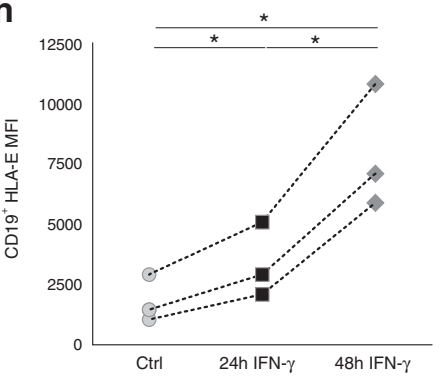

b
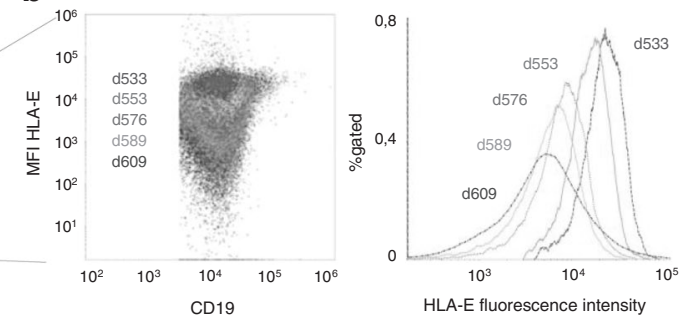

d

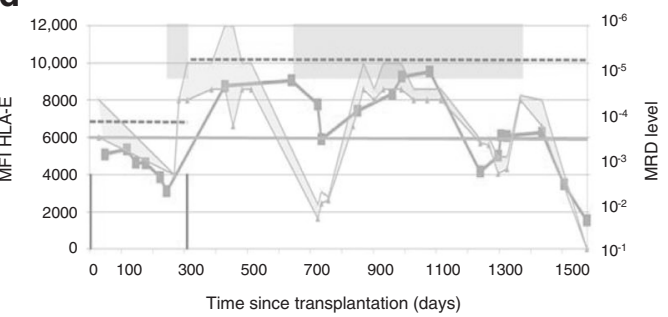

Time since transplantation (days)

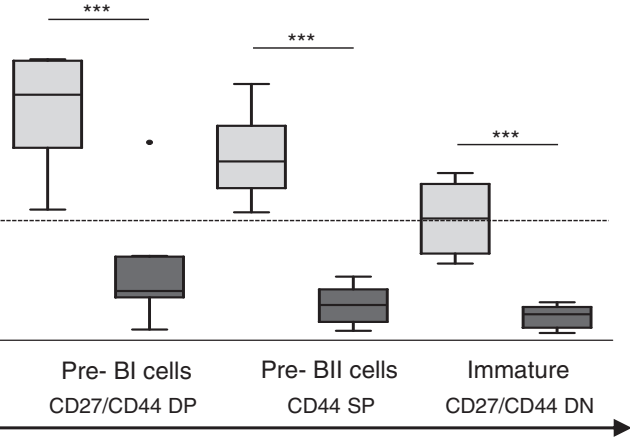

BM
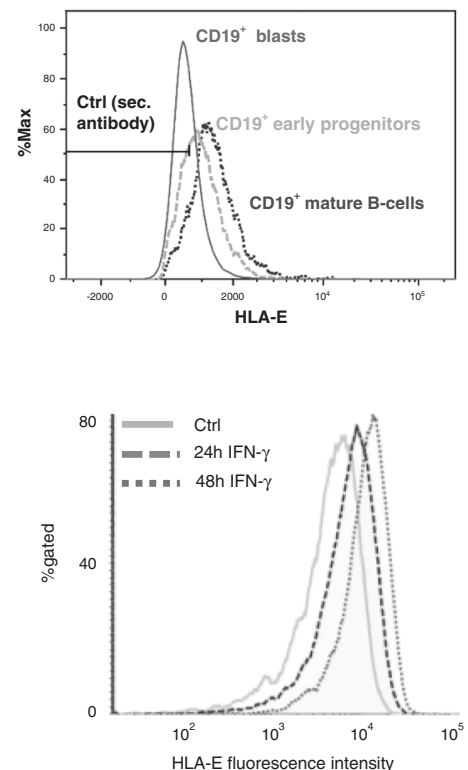

To gain mechanistic insights into the paradoxical finding of HLA-E downregulation, which makes leukemic blasts principally more susceptible to NK cell-mediated killing, functional experiments were performed: blockade of the
NKG2A/HLA-E interaction by using an anti-HLA-E (3D12) $\mathrm{mAb}$ increased the cytolytic activity of healthy NK cells significantly, making leukemic blasts susceptible to recognition by $\mathrm{NKG}_{2} \mathrm{~A}^{+}$NK cells (Fig. $2 \mathrm{~g}$ ). The data 
4 Fig. 2 Leukemic blasts downregulate HLA-E expression to low but tolerogenic levels, which inhibits NK cell activation and correlates with a reoccurrence of the disease. a, c, d Comparison of HLA-E levels and MRD values in three representative patients undergoing multiple allogeneic HSCTs (gray shaded area: range between minimum and maximum detected PCR MRD levels). The three patients were observed for 1.7-4.3 years by analyzing consecutive PBMC samples and results were correlated with analyses of minimal residual disease (MRD) monitoring (PCR and flow-cytometry) in bone marrow samples. Blue shaded area: indicating antineoplastic treatment modalities (chemotherapy, antibody therapy, CIK cell infusion); vertical green: time point of HSCT, red line: HLA-E surface expression; horizontal gray line: PCR MRD threshold; blue dots: flow-cytometry MRD levels; horizontal dashed red line: HLA-E level of the respective donor. b Dot plot (left panel) and histogram (right panel) overlays showing downregulation of HLA-E expression on $\mathrm{CD} 19^{+}$cells from one representative ALL patient at different time points from transplantation to relapse (day +533 violet; day +553 pink; day +576 dark green; day +589 light green; and day +609 blue). e HLA-E surface expression levels of $\mathrm{B}$ cell precursor stages in bone marrow aspirates. Box plots showing staining of HLA-E surface expression on peripheral blood (PB) $\mathrm{CD} 19^{+}$and $\mathrm{CD}^{+}$lymphocytes from 29 healthy volunteers (HV: gray), 40 diagnosed ALL patients (ALL: red, initial and relapse samples) and on bone marrow cells from 8 healthy volunteers (gray) and 8 ALL patients (red). b cell precursor stages in bone marrow aspirates were defined as previously reported in Vaskova et al. [8]. The dotted black baseline indicates the mean expression level of HLA-E on $\mathrm{CD} 19^{+}$peripheral blood cells from healthy volunteers. Error bars represent the standard error of the mean. Statistical significance was determined by a Mann-Whitney $\mathrm{U}$ test or unpaired t-test $(* * * p<$ 0.001). f HLA-E surface expression levels of CD19+ blasts, early progenitors (hematogones) and more mature $\mathrm{CD} 19^{+} \mathrm{B}$ cells. Cell populations and maturity stages were distinguished according to flow MRD standards published by Karawajew et al. [7]. Histogram overlay (right panel) showing downregulation of HLA-E expression on leukemic blasts compared to normal $\mathrm{CD} 19^{+}$cells. $\mathrm{g}$ To further elucidate whether suppression of inhibitory signals could enhance NK cellmediated anti-tumor responses, NKG2A/HLA-E interaction was blocked via $\alpha$ HLA-E (3D12, gray bar). Blocking antibody and cytolytic activity was measured via CD107a mobilization assay. CD107 frequency was measured exclusively on $\mathrm{KIR}^{-} \mathrm{NKG} 2 \mathrm{~A}^{+} \mathrm{CD} 56^{\mathrm{dim}}$ $\mathrm{NK}^{-}$-cells. (h) To determine whether HLA-E downregulation was a reversible process, ALL samples $(n=3)$ were cultured in the presence or absence of IFN $\gamma(100 \mathrm{U} / \mathrm{mL})$ and HLA-E expression was measured at the indicated time points (left panel). The respective histograms of a representative experiment (right panel) at $0 \mathrm{~h}$ (solid violet), $24 \mathrm{~h}$ (dashed blue), and $48 \mathrm{~h}$ (dotted gray). Statistical significance was determined by one-way-Anova or paired t-test $(* p<0.05, * * p<0.01$, $* * * p<0.001)$

demonstrate that the low level of HLA-E, present on ALL blasts, is still sufficient to induce an inhibitory signal through CD94/NKG2A and consequently HLA-E appears to still protect leukemic cells from NK cell-mediated lysis. Importantly, HLA-E downregulation on leukemic blasts could be reversed by IFN $\gamma$ treatment, with full restoration of HLA-E surface expression after $48 \mathrm{~h}$ (Fig. 2g), documenting that the HLA-E gene is not permanently silenced.

Presently, qPCR from bone marrow biopsies represents the most validated and standardized method for MRD detection, which significantly correlates with clinical outcomes in leukemia [9]. The present analyses suggest that flow cytometric quantification of HLA-E expression on leukemic blasts provides a novel promising tool for ALL monitoring in peripheral blood complementing previously established ways to monitor the leukemic burden such as BM-based molecular MRD monitoring. Importantly, no HLA-E deletion variants were detected in any of the ALL samples analyzed so far. Moreover, HLA-E is genetically highly conserved with only two very similar allelic variants (Table S1) [10] that are picked up by the same specific $\mathrm{mAb}$. Based on the data from this and a previous study, together comprising $>50$ different ALL cases, HLA-E constitutes a highly valuable novel flow MRD marker, that is common to all ALL cases analyzed so far and is thus likely to be applicable to virtually all ALL cases. It has to be noted, that the present data have to be validated in a prospective study hopefully leading to an algorithm quantitatively defining critical HLA-E levels that are predictive for MRD. This could provide the basis for a novel strategy in which regular flow cytometric analysis of HLA-E from small blood samples (e.g., $<500 \mu \mathrm{l}$ from finger-prick blood) could potentially improve ALL monitoring by providing a reliable 'alert' signal for subsequent validation by molecular, bone marrow-based MRD assessment.

Mechanistically, the study suggests strong selective pressure for expression of low but still tolerogenic HLA-E levels on ALL blasts, in turn indicating tight surveillance of ALL by $\mathrm{NKG}_{2} \mathrm{~A}^{+} \mathrm{NK}$ cells. The functional data, demonstrating restored killing of ALL blasts by specific blocking of HLA-E/ NKG2A interaction, suggest that HLA-E-mediated resistance to host immune surveillance is only given within a small corridor enabling leukemic escape from both $\mathrm{T}$ cell and NKmediated control. Moreover, they challenge the prevailing view that B-ALL is generally resistant to NK cell surveillance. In fact, cellular therapy combining allogeneic NK cells with antibody-mediated blocking of the HLA-E/NKG2A inhibitory axis, as provided by the NKG2A-specific therapeutic reagent Monalizumab, could open novel avenues for treatment of BALL [11, 12].

Acknowledgements The authors thank all parents who gave their consent to use the biological material from their minors. This work was supported by the DZHK (German Center for Cardiovascular Research, FKZ: $81 \times 2800194$, MU), the Elterninitiative Kinderkrebsklinik e.V. (FB), and the TRANSAID-Stiftung fuer krebskranke Kinder (FB).

Author contributions SBR, ARM, SGK, LK, PAH, and VR performed experiments; $\mathrm{RM}$ and $\mathrm{AB}$ provided samples and conducted data analysis and interpretation and participated in patient care; LK and PAH conducted data analysis and interpretation; SGK and VR performed bioinformatic analyses; SBR, MU, and FB designed and performed bioinformatic analyses; SBR, MU, and FB wrote the manuscript, designed, and directed the study; and all authors contributed to the research and approved the final manuscript.

Funding Open Access funding enabled and organized by Projekt DEAL. 


\section{Compliance with ethical standards}

Conflict of interest The authors declare no competing interests.

Publisher's note Springer Nature remains neutral with regard to jurisdictional claims in published maps and institutional affiliations.

Open Access This article is licensed under a Creative Commons Attribution 4.0 International License, which permits use, sharing, adaptation, distribution and reproduction in any medium or format, as long as you give appropriate credit to the original author(s) and the source, provide a link to the Creative Commons license, and indicate if changes were made. The images or other third party material in this article are included in the article's Creative Commons license, unless indicated otherwise in a credit line to the material. If material is not included in the article's Creative Commons license and your intended use is not permitted by statutory regulation or exceeds the permitted use, you will need to obtain permission directly from the copyright holder. To view a copy of this license, visit http://creativecommons. org/licenses/by/4.0/.

\section{References}

1. Ruggeri L, Capanni M, Urbani E, Perruccio K, Shlomchik WD, Tosti A, et al. Effectiveness of donor natural killer cell alloreactivity in mismatched hematopoietic transplants. Science. 2002;295:2097-100. https://doi.org/10.1126/science.1068440.

2. Pende D, Spaggiari GM, Marcenaro S, Martini S, Rivera P, Capobianco A, et al. Analysis of the receptor-ligand interactions in the natural killer-mediated lysis of freshly isolated myeloid or lymphoblastic leukemias: evidence for the involvement of the Poliovirus receptor (CD155) and Nectin-2 (CD112). Blood. 2005;105:2066-73. https://doi.org/10.1182/blood-2004-09-3548.

3. Feuchtinger T, Pfeiffer M, Pfaffle A, Teltschik HM, Wernet D, Schumm M, et al. Cytolytic activity of NK cell clones against acute childhood precursor-B-cell leukaemia is influenced by HLA class I expression on blasts and the differential KIR phenotype of NK clones. Bone Marrow Transpl. 2009;43:875-81. https://doi. org/10.1038/bmt.2008.398.

4. Pfeiffer M, Schumm M, Feuchtinger T, Dietz K, Handgretinger R, Lang P. Intensity of HLA class I expression and KIR-mismatch determine NK-cell mediated lysis of leukaemic blasts from children with acute lymphatic leukaemia. $\mathrm{Br} \mathrm{J}$ Haematol. 2007;138:97-100. https://doi.org/10.1111/j.1365-2141.2007. 06631.x.

5. Babor F, Manser AR, Fischer JC, Scherenschlich N, Enczmann J, Chazara $\mathrm{O}$, et al. KIR ligand $\mathrm{C} 2$ is associated with increased susceptibility to childhood ALL and confers an elevated risk for late relapse. Blood. 2014;124:2248-51. https://doi.org/10.1182/ blood-2014-05-572065.

6. Reusing SB, Manser AR, Enczmann J, Mulder A, Claas FH, Carrington $\mathrm{M}$, et al. Selective downregulation of HLA-C and HLA-E in childhood acute lymphoblastic leukaemia. Br J Haematol. 2016;174:477-80. https://doi.org/10.1111/bjh.13777.

7. Karawajew L, Dworzak M, Ratei R, Rhein P, Gaipa G, Buldini B, et al. Minimal residual disease analysis by eight-color flow cytometry in relapsed childhood acute lymphoblastic leukemia. Haematologica. 2015;100:935-44. https://doi.org/10.3324/haema tol.2014.116707.

8. Vaskova M, Fronkova E, Starkova J, Kalina T, Mejstrikova E, Hrusak O. CD44 and CD27 delineate B-precursor stages with different recombination status and with an uneven distribution in nonmalignant and malignant hematopoiesis. Tissue Antigens. 2008;71:57-66. https://doi.org/10.1111/j.1399-0039.2007.00968.x.

9. Shahkarami S, Mehrasa R, Younesian S, Yaghmaie M, Chahardouli B, Vaezi M, et al. Minimal residual disease (MRD) detection using rearrangement of immunoglobulin/T cell receptor genes in adult patients with acute lymphoblastic leukemia (ALL). Ann Hematol. 2018;97:585-95. https://doi.org/10.1007/s00277018-3230-z.

10. Geraghty DE, Stockschleader M, Ishitani A, Hansen JA. Polymorphism at the HLA-E locus predates most HLA-A and -B polymorphism. Hum Immunol. 1992;33:174-84.

11. McWilliams EM, Mele JM, Cheney C, Timmerman EA, Fiazuddin F, Strattan EJ, et al. Therapeutic CD94/NKG2A blockade improves natural killer cell dysfunction in chronic lymphocytic leukemia. Oncoimmunology. 2016;5:e1226720. https://doi.org/ 10.1080/2162402x.2016.1226720.

12. Andre P, Denis C, Soulas C, Bourbon-Caillet C, Lopez J, Arnoux $\mathrm{T}$, et al. Anti-NKG2A mAb is a checkpoint inhibitor that promotes anti-tumor immunity by unleashing both $\mathrm{T}$ and NK cells. Cell. 2018;175:1731-1743 e1713. https://doi.org/10.1016/j.cell.2018. 10.014 . 